\title{
Desenvolvimento morfológico das larvas de Hoplias lacerdae Miranda Ribeiro, 1908 (Characiformes, Erythrinidae), da eclosão até a metamorfose, relacionado com a capacidade de capturar alimento exógeno
}

\author{
Cláudia Maria Reis Raposo Maciel ${ }^{1 *}$ \\ Alaor Maciel Júnior ${ }^{2}$ \\ Juarez Lopes Donzele ${ }^{3}$ \\ Eduardo Arruda Teixeira Lanna ${ }^{3}$ \\ Eliane Menin ${ }^{4}$ \\ ${ }^{1} \mathrm{DEBI} / \mathrm{UESB}$, Universidade Estadual do Sudoeste da Bahia \\ CEP 45.700-000, Itapetinga - BA, Brasil \\ ${ }^{2}$ DTRA/UESB, Universidade Estadual do Sudoeste da Bahia, Itapetinga - BA, Brasil \\ ${ }^{3}$ DZO/UFV, Universidade Federal de Viçosa, CEP 36.571-000, Viçosa - MG, Brasil \\ ${ }^{4} \mathrm{DBA} / \mathrm{UFV}$, Universidade Federal de Viçosa, Viçosa - MG, Brasil \\ *Autor para correspondência \\ crmaciel@uesb.br
}

\section{Resumo}

Para a realização deste trabalho foram utilizados 510 exemplares de Hoplias lacerdae, com um a 30 dias após a eclosão, com o objetivo de estudar as características morfológicas, mesoscópicas, que capacitam essa espécie, nas fases iniciais do seu desenvolvimento, a apresentarem estruturas adaptadas para se locomoverem e capturarem alimento exógeno. As larvas vitelínicas com 11 dias após a eclosão têm potencialidade para capturar alimento exógeno, ou seja, presas, mesmo antes da absorção total do vitelo, o que acontece com 12 dias. Nesta fase apresentam: boca aberta com um dia após a eclosão e posição terminal aos quatro dias; aos sete dias demonstram flexão da notocorda, proporcionando maior eficiência locomotora, bem como apresenta neste período nadadeiras peitorais desenvolvidas, as quais facilitam o equilíbrio e direcionamento na coluna d'água; e aos oito dias visualizou-se a formação das nadadeiras dorsal e anal, porém as nadadeiras pélvicas foram visualizadas aos 11 dias.

Unitermos: alimentação, morfologia, ontogênese, Teleostei, trairão

\section{Abstract}

Larval morphology of Hoplias lacerdae Miranda Ribeiro, 1908 (Characiformes, Erythrinidae), from hatching to metamorphosis, related to exogenous food capture ability. For the accomplishment of this work, 510 specimens of Hoplias lacerdae were used from one to 30 days after hatching, with the objective of studying the mesoscopic morphological characteristics that enable this species, in the initial phases of its development, 
to present adaptations for movement and the capture of exogenous food. The yolk sac larvae at 11 days after hatching have the potentiality to capture exogenous food, i.e. prey, even before the total yolk absorption which happens at 12 days, presenting in this phase: an open mouth at one day after hatching, displaying the terminal position at four days; notochord flexion, that takes place at seven days and provides greater efficiency of movement; pectoral fins, also developed at seven days, which facilitate its balance and direction in the water column; formation of the dorsal and anal fins at eight days and the pelvic ones, at 11 days.

Keywords: feeding, morphology, ontogenesis, Teleostei, trairão

\section{Introdução}

A descrição do aparecimento e desenvolvimento de características corporais externas é parte do estudo da morfologia de larvas, importante para compreender o surgimento de estruturas com as respectivas ordens cronológicas, que permitem ao animal uma maior autonomia de movimentação, alimentação e percepção do meio, e, consequentemente, proporcionam maior sobrevivência no meio em que se encontram (Maciel, 2006). A maioria dos morfologistas funcionais acredita que cada aspecto morfológico de um organismo e a sua função têm sido moldados pela seleção natural, como uma solução, ou seja, uma adaptação para um problema apontado pelo ambiente. Na maioria dos estudos, a meta da análise morfológica funcional é revelar uma adaptação particular (Liem, 1980).

Os aspectos morfológicos relacionados à nutrição de peixes com potencialidade comercial são de extrema importância, principalmente nas fases iniciais do seu ciclo de vida, uma vez que a falta de informações acerca da morfologia e fisiologia digestiva pode levar ao manejo nutricional inadequado nessas fases podendo comprometer o desempenho futuro dos animais em cativeiro.

As espécies da família Erythrinidae (Ordem Characiformes), conhecidas como "traíras", "trairões", "jejus" e "marobás", são peixes de água doce, que se distribuem nas regiões tropicais e subtropicais da América do Sul e Central, são carnívoros de hábitos sedentários e ocorrem em vários tipos de ambientes fluviais e lacustres (Paiva, 1974; Nakatani et al., 2001). O conjunto de características morfológicas que define a família é: presença de dentes cônicos e caninos nas maxilas e dentículos cônicos no palato; nadadeira adiposa e fontanela frontal sempre ausentes (Britski et al., 1988).
O gênero Hoplias distingue-se, dos outros gêneros da família, por apresentar, dentes caninos no maxilar e na porção anterior e posterior do dentário (Britski et al., 1988). Este gênero possui situação taxonômica bastante confusa, em razão da grande quantidade de espécies descritas e do uso de características inadequadas para a sua delimitação (Oyakawa, 1993).

Os trairões, H. lacerdae, possuem a localidadetipo na Bacia do Ribeira do Iguape, estado de São Paulo (Buckup et al., 2007; Menezes et al., 2007) e, aparentemente, não estão ameaçados ou em perigo de extinção (Menezes et al., 2007). Pelo porte, apresentam grande potencial de cultivo, sendo, ainda, muito apreciados na pesca desportiva e na culinária (Neves, 1996). Esta espécie tem sido utilizada no peixamento de vários reservatórios, uma vez que apresenta algumas características que a indicam para a criação comercial, tais como: facilidade de desovar naturalmente em cativeiro, isto é, em condições de laboratório; alta prolificidade, permitindo boa disponibilidade de alevinos; elevada taxa de ganho de peso e sedentarismo (Gontijo, 1984).

Este trabalho teve por finalidade estudar as características morfológicas aparentes que capacitam a espécie H. lacerdae, nas fases iniciais do seu desenvolvimento até a metamorfose, a apresentarem as adaptações para se locomoverem e capturarem alimento exógeno.

\section{Material e Métodos}

As larvas de Hoplias lacerdae (Miranda-Ribeiro, 1908), provenientes da Estação de Hidrobiologia e Piscicultura do Departamento de Biologia Animal da Universidade Federal de Viçosa, Viçosa, Minas Gerais, foram obtidas por desova natural nos tanques de reprodução da referida estação. Depois de coletados, os ovos foram transferidos para uma incubadora de 50L 
presente no Laboratório de Incubação da referida Estação e estes foram mantidos por sete dias, a $28^{\circ} \mathrm{C} \mathrm{e} \mathrm{pH} \mathrm{de} \mathrm{7,16.}$ As coletas foram feitas, diariamente, por sifonagem, com auxílio de uma mangueira plástica transparente.

Após sete dias, as larvas foram transferidas para um tanque de terra de $400 \mathrm{~m}^{2}$, de acordo com técnicas de rotina da Estação, e, diariamente, durante 30 dias, foram coletados de 12 a 18 exemplares, com o auxílio de redes de malhas finas.

Os exemplares coletados foram sedados por hipotermia (conforme Resolução ${ }^{0}{ }^{714}$, de 20/07/2002 do Conselho Federal de Medicina Veterinária) e posteriormente, foram fixados em solução aquosa de formaldeído neutro tamponado, a 4\%, e após 8 a 12h, transferidos para solução de álcool a $70 \%$.

As análises morfológicas dos exemplares em estudo foram realizadas no Laboratório de Morfofisiologia Animal do Departamento de Biologia Animal, CCB/ UFV, Viçosa, MG. Essas análises consistiram na verificação mesoscópica das características externas do corpo dos exemplares, tais como: aparecimento e localização da pigmentação no corpo (presença e tipos de cromatóforos), deslocamento da fenda bucal para a posição terminal e abertura da boca (diferenciação da boca), formação das nadadeiras, formação dos arcos branquiais e do opérculo, flexão da notocorda e redução do saco vitelino (Maciel Junior, 1996). Para complementar essas análises com ilustrações, foi utilizado um microscópio estereoscópico Olympus SZH10 Research Stereo, modelo 131, com aumento máximo de 70 vezes, equipado com câmara clara.

As análises relativas à morfometria dos exemplares, ou seja, comprimento-total e comprimento-padrão, foram realizadas com um paquímetro digital Mytutoyo, modelo Digimatic Caliper, com precisão de $0,001 \mathrm{~mm}$, e com o auxílio do microscópio estereroscópico descrito anteriormente. $\mathrm{O}$ comprimento-total corresponde à distância entre a extremidade rostral da cabeça e a extremidade caudal da nadadeira embrionária das larvas (Figuras 1A e 1B) e o comprimento-padrão, à distância entre a extremidade rostral da cabeça e o pedúnculo da nadadeira caudal dos alevinos (Figura 1C) (Ricker, 1968; Maciel Júnior, 1996; Nakatani et al., 2001).

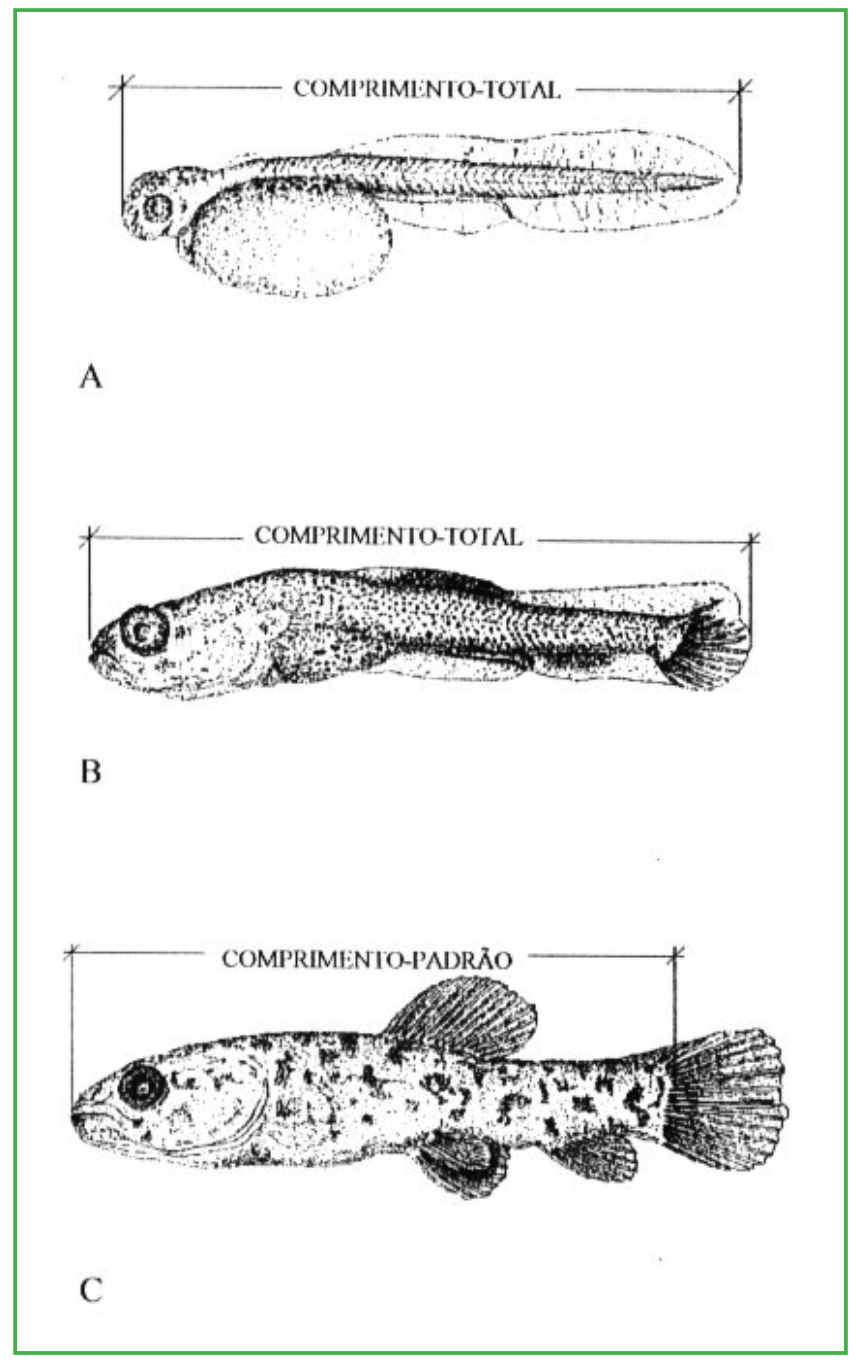

FIGURA 1: Medidas morfométricas realizadas em Hoplias lacerdae, trairão. Comprimento-total das larvas vitelínicas (A) e das larvas (B) e comprimento-padrão dos alevinos (C) (Figura de Maciel Júnior).

\section{Resultados e Discussão}

As larvas vitelínicas de Hoplias lacerdae (trairão), com um dia após a eclosão, medem $6,35+0,19 \mathrm{~mm}$ de comprimento-total e apresentam-se transparentes, com a pigmentação mais concentrada na região dorsal da cabeça e nos olhos (Figura 2A). A pigmentação do saco vitelino apresenta-se mais intensa na região dorsolateral. Os miômeros são visíveis, assim como as nadadeiras peitorais, que se localizam na região dorsolateral ao saco vitelino. A nadadeira embrionária apresenta-se ricamente vascularizada. A boca ventral, em formação, encontra-se aberta, mas não-funcional, e localiza-se anteriormente à 
face frontal da cavidade pericárdica, que é transparente e disposta, por sua vez, anteriormente à extremidade cranial do saco vitelino. $\mathrm{O}$ saco vitelino envolve uma massa granulosa de vitelo de coloração amarelada, de grande volume, de forma, mais frequentemente, elipsóide. $\mathrm{O}$ tubo digestivo está completo, mas não funcional e, em nível mesoscópico, é longo, retilíneo, de luz estreita e, na região posterior, curva-se dorsoventralmente, desembocando, posteriormente, no saco vitelino, local em que se formará o ânus. A bexiga gasosa é observada como uma estrutura refringente, de pequeno tamanho e de forma elíptica, disposta na região dorsocaudal em relação ao saco vitelino.

Aos dois dias após a eclosão, as larvas vitelínicas de H. lacerdae medem $8,04+0,22 \mathrm{~mm}$ de comprimentototal, a pigmentação é mais intensa na cabeça e no corpo, formando uma faixa estreita dorsal e outra na região lateral do saco vitelino. Os miômeros, sob a luz incidente, ainda são visíveis. As nadadeiras peitorais estão mais bem desenvolvidas. A boca possui posição semiventral e o tubo digestivo encontra-se mais nítido que no dia anterior. $\mathrm{O}$ saco vitelino apresenta ligeira redução na porção anteroventral e esboços de arcos branquiais podiam ser encontrados.

As larvas vitelínicas de $H$. lacerdae, com três dias após a eclosão, medem $8,75+0,27 \mathrm{~mm}$ de comprimentototal e apresentam a pigmentação dos olhos completa; o corpo com pigmentação uniformemente distribuída, com exceção da cabeça, região em que ela era mais concentrada, e da região ventral do saco vitelino, em que ela era menos concentrada. A boca passa a ocupar a posição terminal, característica da espécie, com os dentes orais tornando-se visíveis, mesmo sem técnicas específicas. A luz do tubo digestivo se estreita e com o ânus já formado. Nessa fase, cerca de 40 a $50 \%$ do vitelo já foi consumido, estando o saco vitelino, agora, mais volumoso na porção caudal.

Nas larvas vitelínicas de $H$. lacerdae, com cinco dias após a eclosão (Figura 2B) e $8,79 \pm 0,24 \mathrm{~mm}$ de comprimento-total, o vitelo apresenta-se reduzido, ou seja, aproximadamente mais de $60 \%$ já tinha sido absorvido. Começam a surgir os raios mesenquimais da nadadeira caudal na região caudoventral da notocorda. Os arcos branquiais estão em desenvolvimento. A larva passa a morfologia de alevino, com corpo mais fusiforme. Aos seis dias após a eclosão, medindo 9,22+0,25mm de comprimento-total, o vitelo das larvas dessas larvas está mais reduzido, mais de $80 \%$ em relação ao observado no dia anterior. Ocorre um início da flexão da notocorda e a nadadeira caudal está em início de formação. As larvas apresentam-se mais pigmentadas, sob a luz transmitida, inviabilizando a verificação dos órgãos internos sem técnicas específicas.

A notocorda das larvas vitelínicas de H. lacerdae, aos sete dias após a eclosão, continua em processo de flexão, na sua extremidade caudal, em sentido dorsal, o que colocam os raios mesenquimais da nadadeira caudal, em formação, na sua posição definitiva, ou seja, longitudinal. A luz do tubo digestivo apresenta-se maior em alguns pontos, que pode representar o início da diferenciação do estômago.

As larvas vitelínicas com oito dias após a eclosão, a notocorda apresenta-se flexionada, a nadadeira caudal em processo de formação e o vitelo, quase todo consumido (Figura 2C). Pode-se observar espessamentos na nadadeira embrionária, um na região dorsal, que se inicia na altura da extremidade caudal do saco vitelino e chega até o terço final do tubo digestivo, e outro na região ventral dessa nadadeira, que se inicia logo após a região caudal do tubo digestivo e atinge o meio da região compreendida entre o ânus e o início do pedúnculo da nadadeira caudal. Esses espessamentos, na nadadeira embrionária, correspondem à formação das nadadeiras dorsal e anal, respectivamente. As nadadeiras peitorais estão pigmentadas, e os arcos branquiais bem desenvolvidos. Essas larvas medem agora 10,01+0,37mm de comprimento-total (Figura 2C).

Aos nove dias após a eclosão, as larvas vitelínicas de H. lacerdae medem 10,79+0,16mm de comprimentototal. Com 10 dias após a eclosão e $11,16+0,73 \mathrm{~mm}$ de comprimento-total, a sua pigmentação torna-se acentuada, inviabilizando, totalmente, a observação de características internas dos exemplares, por meio de luz transmitida. Os raios das nadadeiras dorsal e anal estão evidentes. 


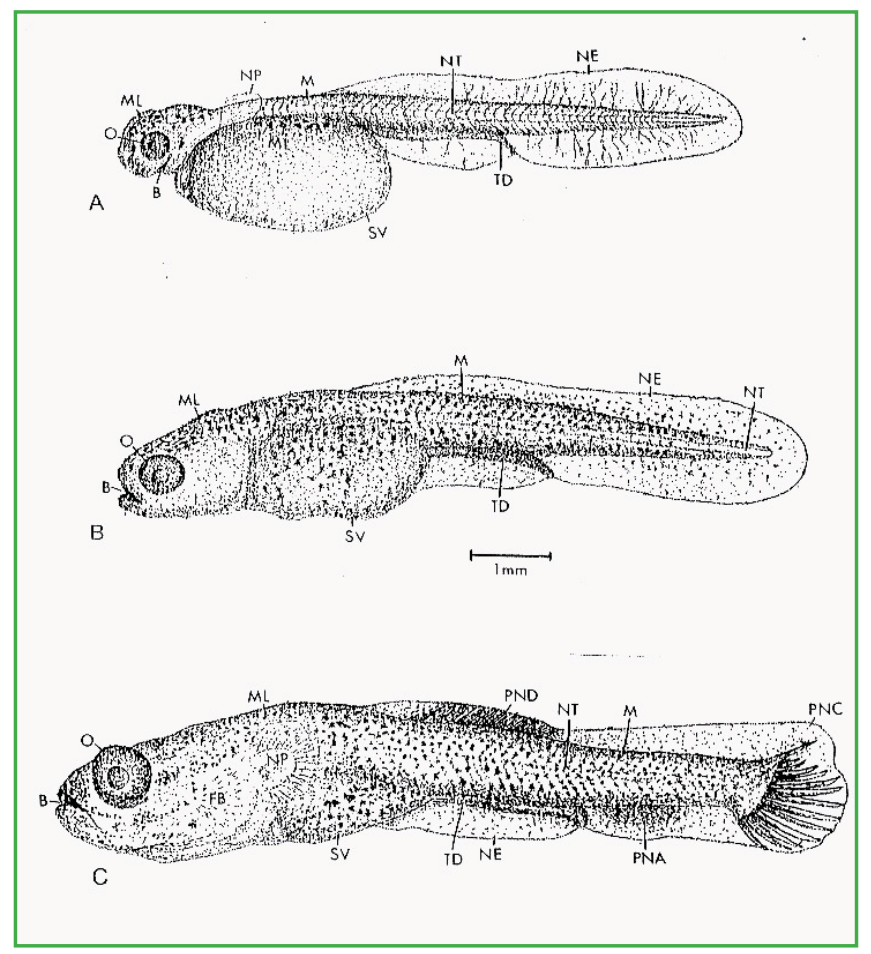

FIGURA 2: Vista lateral esquerda de larvas vitelínicas de Hoplias lacerdae, trairão, com um dia (A), cinco dias (B) e oito dias (C) após a eclosão. B - boca; FB - filamentos branquiais; $\mathrm{M}$ - miômeros; $\mathrm{ML}$ - melanóforos; PNA - primórdio da nadadeira anal; PNC - primórdio da nadadeira caudal; PND - primórdio da nadadeira dorsal; NE - nadadeira embrionária; NP - nadadeira peitoral; NT - notocorda; O - olho; SV - saco vitelino; $\mathrm{TD}$ - tubo digestivo.

Aos 11 dias após a eclosão, as larvas vitelínicas da espécie em questão medem $12,10+0,23 \mathrm{~mm}$ de comprimento-total. A nadadeira embrionária está mais reduzida, evidenciando as nadadeiras dorsal e anal, e as nadadeiras pélvicas iniciavam sua formação. O vitelo encontra-se reduzido, porém, ainda, passível de diferenciação em nível mesoscópico.

Aos 12 dias após a eclosão, os exemplares de $H$. lacerdae apresentam características de larvas e medem $13,07+0,18 \mathrm{~mm}$ de comprimento-total. A nadadeira embrionária continua em processo de redução e, nas nadadeiras peitorais, caudal, dorsal e anal, a pigmentação está acentuada e segue o contorno de seus respectivos raios. $\mathrm{O}$ vitelo não pode mais ser identificado em nível mesoscópio, pois se confunde com outras estruturas, necessitando preparação histológica para definir seus limites.
Aos 13 dias após a eclosão, nas larvas de $H$. lacerdae a pigmentação começa a se concentrar em alguns pontos do corpo, como na região frontal da cabeça, e a nadadeira embrionária está mais reduzida na região dorsal que na ventral. Essas larvas medem $13,28+0,33 \mathrm{~mm}$ de comprimento-total (Figura 3A).

Aos 14, 15, 17 e 18 dias após a eclosão, as larvas de $H$. lacerdae com $14,83+0,51 \mathrm{~mm}, 14,84+0,62 \mathrm{~mm}$, $16,52+0,26 \mathrm{~mm}$ e $16,58+0,95 \mathrm{~mm}$ de comprimentototal, respectivamente, e com os raios das nadadeiras formados, ou seja, o desenvolvimento das nadadeiras é o evento marcante nesse período. Aos 17 dias, pode ser observado que a pigmentação das larvas concentrase em várias regiões do corpo, formando manchas inconspícuas, que podem ser notadas, até mesmo a olho nu. As larvas tornam-se semelhantes aos adultos, embora a nadadeira embrionária ainda está presente.

Aos 19 dias após a eclosão, os exemplares de H. lacerdae apresentam características de alevinos e assemelham-se aos adultos, medindo $15,25+0,44 \mathrm{~mm}$ de comprimento-padrão, e passam a ser denominadas "alevinos". A nadadeira embrionária desapareceu completamente e as nadadeiras peitorais, pélvicas, caudal, dorsal e anal estão formadas.

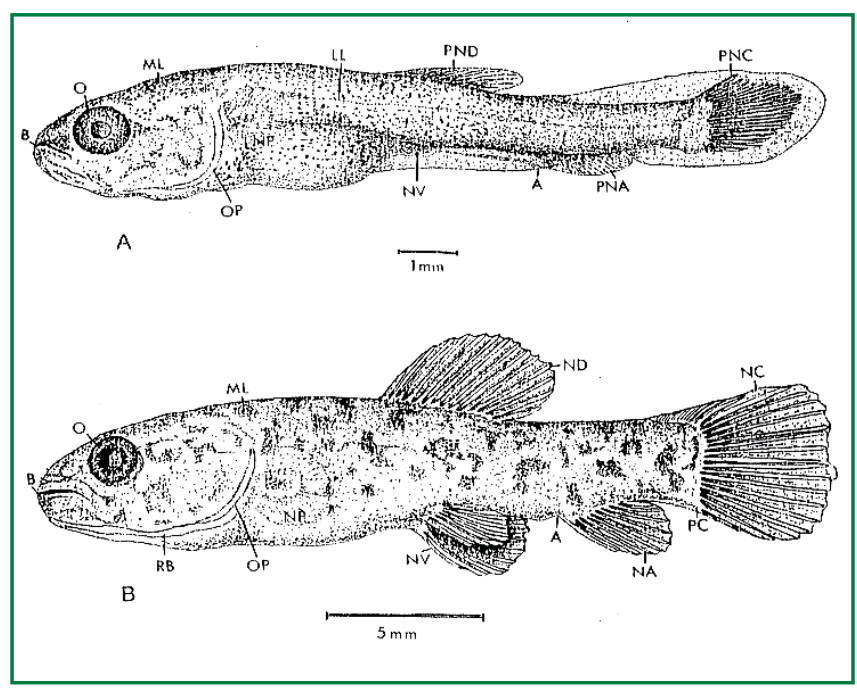

FIGURA 3: Vista lateral esquerda de larvas de Hoplias lacerdae (trairão), no 13 dia após a eclosão (A) e alevinos, no 24ํ dia após a eclosão (B). A - ânus; B - boca; LL - linha lateral; ML - melanóforos; NA - nadadeira anal; $\mathrm{NC}$ - nadadeira caudal; ND - nadadeira dorsal; $\mathrm{NP}$ - nadadeira peitoral; NV - nadadeira ventral; O - olho; OP - opérculo; PC - pedúnculo caudal; PNA - primórdio da nadadeira anal; PNC - primórdio da nadadeira caudal; PND - primórdio da nadadeira dorsal; $\mathrm{RB}$ - raios branquiostegais. 
Entre 20 a 30 dias após a eclosão, nos alevinos, a pigmentação é o evento marcante, ou seja, ela constitui um padrão definido, que será evidenciado inclusive nas fases juvenil e adulta. Nos dias 21, 23, 24 (Figura 3B), 25, 28 e 30, os alevinos medem $16,46+1,08 \mathrm{~mm}$, $17,99+0,62 \mathrm{~mm}, 20,65+0,90 \mathrm{~mm}, 21,47+0,45 \mathrm{~mm}$, $24,21+1,08 \mathrm{~mm}$ e $31,87+0,43 \mathrm{~mm}$, respectivamente, de comprimento-padrão.

A síntese dos principais eventos morfológicos registrados durante a ontogênese das larvas de $H$. lacerdae está sintetizado na Tabela 1.

TABELA 1: Síntese dos principais eventos morfológicos no desenvolvimento das larvas de Hoplias lacerdae.

\begin{tabular}{cl}
\hline $\begin{array}{c}\text { Dias após a } \\
\text { eclosão }\end{array}$ & \multicolumn{1}{c}{ Eventos Morfológicos } \\
\hline 01 & Abertura da boca, boca ventral \\
02 & boca semiventral \\
03 & olhos pigmentados, dentes orais \\
04 & boca terminal \\
05 & arcos branquiais desenvolvidos \\
07 & início da flexão da notocorda, nadadeiras \\
& peitorais pigmentadas \\
08 & início da formação das nadadeiras dorsal e \\
& anal \\
10 & início da formação dos raios nadadeiras dor- \\
11 & sal e anal \\
12 & início da formação das nadadeiras pélvicas \\
17 & ausência de vitelo \\
19 & nigmentação forma manchas \\
20 & pigmentação inconspícua \\
\hline
\end{tabular}

Woynarovich e Horváth (1989) verificaram que as larvas recém-eclodidas são diferentes dos peixes adultos, por não apresentarem boca, intestino, ânus, brânquias e bexiga gasosa, e a maioria inclusive a pigmentação ou com ela muito esparsa e diferindo, em padrão, entre as espécies. Segundo Nakatani et al. (2001), as larvas de H. lacerdae eclodem às $35 \mathrm{~h} 30 \mathrm{~min}$ após a fecundação, à temperatura de $27,1^{\circ} \mathrm{C}$, medindo cerca de $5,53 \mathrm{~mm}$ comprimento-padrão, tendo o saco vitelino relativamente grande, os olhos pouco pigmentados e não apresentam pigmentação aparente no corpo. Maciel (2006) relatou que nas larvas de Brycon orbignyanus (piracanjuba), a pigmentação dorsal aumentou significativamente nas primeiras $24 \mathrm{~h}$ após a eclosão.

Para Bone et al. (1995), a despigmentação das larvas recém-eclodidas é uma estratégia para o escape de predação, comum à maioria das larvas de Teleostei, e segundo Costa et al. (2002), é uma forma de camuflagem das larvas no seu período mais vulnerável, como também às características taxonômicas (Santos e Godinho, 1996; 2003).

Os pigmentos das larvas de trairão referemse, possivelmente, à melanina e os cromatóforos, consequentemente, à melanóforos, cujos limites celulares são indiferenciados. Essas células, com coloração escura, em razão do pigmento, são encontradas dispersas na superfície corpórea; a maioria delas tem forma dendrítica e, com os grânulos de pigmentos dispersos, tomam forma estrelada e, ou retículo-estrelada, o que caracteriza os graus 3 e 4, conforme o Índice Melanofórico, de cinco graus, de Hogben e Slome (1931, apud Hoar e Hickman, 1967), para os cromatóforos de anfíbios.

A coloração dos cromatóforos no trairão é semelhante à descrita no trabalho de Allison e Rojas (1975) para Locariichthys typus. Segundo Chirinos de Vildoso e Chuman (1964, apud Santos, 1992), o número de cromatóforos não é constante para a espécie e aumenta com a idade. Meijide e Guerrero (2000) e Godinho et al. (2003) ressaltaram que uma descrição pormenorizada de cromatóforos de larvas de peixes é importante, pois eles são um importante caráter taxonômico utilizado em identificação de espécies. Segundo Santos (1992), tal critério pode ser aplicado especialmente para as larvas de Piaractus mesopotamicus (pacu), Leporinus elongatus (piau verdadeiro) e Pseudoplatystoma coruscans (surubim), dentre as espécies por ele estudadas.

Segundo Pinder e Gozlan (2004), a abertura da boca acontece concomitantemente com a capacidade do exemplar nadar até a superfície e começar a tragar o ar para inflar a bexiga gasosa.

Neves (1996) relatou que, embora a boca estivesse aberta, em larvas de $H$. lacerdae, incubadas a $28^{\circ} \mathrm{C}$, no primeiro dia após a eclosão, o esôfago mostrou-se luminado e com características anatômicas próprias somente no terceiro dia. Entretanto, Sirol (1995) 
verificou a abertura da boca nas larvas dessa espécie, incubadas a $26,5^{\circ} \mathrm{C}$, somente 2,5 dias após a eclosão, e que elas se apresentavam despigmentadas até o segundo dia após a eclosão. Grizzle e Curd (1978) e Walford e Lam (1993) observaram a abertura da boca de Percina caprodes (perca) e Lates calcarifer (seabass), com três e dois dias de idade após a eclosão, respectivamente. A abertura e o tamanho da boca permitem a inferência a respeito do tamanho do alimento que a larva é capaz de ingerir (Bone et al., 1995).

Conforme Santos e Godinho (1996; 2002), o aparecimento da boca e a pigmentação da retina ocorrem simultaneamente e estão relacionados à primeira alimentação.

De acordo com Souza (1999), as maiores alterações do aparelho digestório ocorrem durante o período de desenvolvimento larval. Este autor relatou que o aparecimento de dentículos orais capacita o animal para uma preensão mais eficiente do alimento. Segundo Gisbert et al. (2002), o desenvolvimento de estruturas de alimentação, como uma mandíbula funcional, é determinante para a captura da presa, o que proporciona o crescimento larval e suas chances de sobrevivência. A presença de alimentos ao longo do tubo digestivo de $H$. lacerdae foi observada por Neves (1996) em exemplares com 12 dias de idade.

O desenvolvimento da estrutura branquial é importante para a capacitação do animal à natação, visto que a troca gasosa se torna mais efetiva em comparação à respiração cutânea da fase larval (Bone et al., 1995).

De acordo com Matkovic e Pisanó (1989), larvas de H. malabaricus (traíra), ao eclodirem, já apresentam os esboços das nadadeiras peitorais, que também foram observadas em H. lacerdae e, segundo Santos (1992), o desenvolvimento dessas nadadeiras é uma aquisição importante na organogênese das larvas de peixes, uma vez que essas estruturas facilitam o equilíbrio e o direcionamento na coluna d'água. Machinandiarena et al. (2003) verificaram que o aparecimento das nadadeiras coincidiu com a transformação das larvas pelágicas de Pagrus pagrus (red porgy), entretanto, Carvalho (2001) não observou esboços de nadadeiras peitorais em larvas recém eclodidas de Salminus maxillosus (dourado).
Diferentemente do que foi observado em trairão, Chacon (1975) verificou, em larvas de Trachycorystes galeatus (cangati catfish), com dois dias após a eclosão, que as primeiras indicações dos raios das nadadeiras caudais e que, aos cinco dias, as nadadeiras peitorais estavam desenvolvidas.

Chacon (1975) constatou, na espécie por ele estudada, Trachyrorystes galeatus que as nadadeiras estão definitivamente formadas aos 13 dias após a eclosão e que, nessa espécie, as larvas, após a eclosão, parecem mais desenvolvidas que as de Cichlidae e Prochilodontidae.

Para Dasilao Jr. et al. (2002), uma nadadeira caudal heterocerca confere um grau de alta estabilidade para as larvas, em particular, durante a natação. Esses mesmos autores relataram que, durante a ontogenia, várias mudanças morfológicas melhoram a propulsão caudal, como a região caudal que fica relativamente mais longa e delgada e, desta forma, mais ágil.

Nakatani et al. (2001) observaram que a absorção completa do saco vitelino, em trairão, ocorre com cerca de $9,30 \mathrm{~mm}$ de comprimento padrão (flexão). O período de absorção do saco vitelino é vital para a larva, pois nesse período, o aparelho digestório ainda se encontra em diferenciação (Godinho et al., 2003). Segundo Blaxter (1988), após a absorção do vitelo, a larva deverá apresentar boca funcional e intestino e olhos desenvolvidos, para que possa buscar fontes nutricionais exógenas, como plâncton e, ou partículas orgânicas.

Santos (1992) e Godinho et al. (2003) relataram que, nas seis espécies por ele estudadas, o tempo de duração do saco vitelino não foi uniforme. Essa estrutura, nas larvas de Salminus brasiliensis (dourado), teve absorção quase total no terceiro dia; nas larvas de Prochilodus affinis (curimatá-pioa), Prochilodus marggravii (curimatápacu) e Pseudoplatystoma coruscans (surubim), no quarto dia; nas larvas de Leporinus elongatus (piauverdadeiro), no quinto dia; e nas larvas do Piaractus mesopotamicus (pacu), no sexto dia.

A flexão da notocorda da larva determina o início da formação da nadadeira caudal, o que proporciona e favorece maior eficiência da natação e da captura de presas, tanto em qualidade quanto em quantidade, além 
de apresentar maior agilidade na fuga dos predadores (Blaxter, 1988). Sirol (1995) relatou que a flexão da notocorda, nas larvas de $H$. lacerdae, incubadas a $26,5^{\circ} \mathrm{C}$, tem inicio aos quatro dias após a eclosão e finaliza aos 6,5 dias.

Segundo Boulhic e Gabaudan (1992), em Solea solea (dover solea), diferentemente do observado em H. lacerdae, o período larval vai do segundo ao $22^{\circ}$ dia após a eclosão, e a mais importante mudança acontece na fase de vitelo: a abertura da boca permite que as necessidades tróficas sejam providas por suprimentos endógenos e exógenos.

Verificamos que as larvas de trairão, H. lacerdae, a partir de 11 dias após a eclosão desenvolveram um conjunto de características morfológicas que as capacitam para capturar alimento exógeno, uma vez que apresentam boca aberta e terminal, notocorda flexionada, nadadeiras peitorais, dorsal, anal e pélvicas desenvolvidas, e para superar uma das etapas mais críticas da larvicultura. Assim conclui-se que as estruturas analisadas, em conjunto, favorecem a natação e possibilitam a captura de alimento, a busca de ambiente favorável e o escape de predadores.

\section{Referências}

Allison, A. M.; Rojas, H. L. 1975. Etapas del desarrolo de Loricariichthys typus Bleeker, 1964 (Osteichthyes, Siluriformes, Loricariidae). Acta Biológica Venezuelica, 9 (1): 93-119.

Blaxter, J. H. S. 1988. Pattern and variety in Development. In: Hoar, W. S. \& Randall, D. J. (Eds). Fish physiology. v.11a. Academic Press, San Diego, USA, p.1-48.

Bone, Q.; Marshall, N. B.; Blaxter, J. H. S. 1995. Biology of Fishes. $2^{\text {nd }}$ ed. Blackie Academic \& Professional, London, UK, 332pp.

Boulhic, M.; Gabaudan, J. 1992. Histological study of the organogenesis of the digestive system and swim bladder of the Dover solea, Solea solea (Linnaeus 1758). Aquaculture, 102: 373-396.

Britski, H. A.; Sato, Y.; Rosa, A. B. S. 1988. Manual de identificação de peixes da região de Três Marias. $3^{\mathrm{a}}$ ed. Câmara dos Deputados, Coordenação de Publicações/ CODEVASP, Divisão de Piscicultura e Pesca, Brasília, Brasil, 115pp.

Buckup, P. A.; Menezes, N. A.; Ghazzi, M. S. 2007. Catálogo das espécies de peixes de água doce do Brasil. Museu Nacional, Rio de Janeiro, Brasil, 195pp.

Carvalho, F. 2001. Desenvolvimento do aparelho digestório em larvas de Salminus maxillosus Valenciennes, 1849 (Characiformes, Characidae, Salmininae). Monografia, Universidade Federal de Viçosa, Brasil, 59pp.
Chacon, J. O. 1975. Embryonic and early larval stages of "Cangati Catfish", Trachycorystes galeatus Linnaeus, 1756, at the amanari fish culture station, Maranguape, Ceará, Brazil. Revista Brasileira de Biologia, 35 (4): 737-744.

Costa, G. C.; Menin, E.; Oliveira, A. L. S. de. 2002. Morfologia aparente de larvas de Paulicea Luetkeni (Steindachner, 1875) na fase inicial de desenvolvimento (Siluriformes, Siluroidei, Pimeloididae). Resumos do XII Simpósio de Iniciação Científica da UFV, Viçosa, Brasil, p.53.

Dasilao Jr., J.; Rossiter, A.; Yamaoka, K. 2002. Adaptive ontogenetic shape change in flyingfish Parexocoetus mento mento. Fisheries Science, 68 (1): 71-76.

Gisbert, E.; Merino, G.; Muguet, J. B.; Bush, D.; Piedrahita, R. H.; Conklin, D. E. 2002. Morphological development and growth patterns in hatchery-read Califórnia halibut larvae. Journal of Fish Biology, 61: 1217-1229.

Godinho, H. P.; Santos, J. E.; Sato, Y. 2003. Ontogênese larval de cinco espécies de peixes do São Francisco. In: Godinho, H. P. \& Godinho, A. L. (Orgs). Águas, peixes e pescadores do São Francisco das Minas Gerais. PUCMG, Belo Horizonte, Brasil, p.133-148

Gontijo, V. P. M. 1984. Produção consorciada de trairão e tilápia. Informe Agropecuário, 10 (110): 26-29.

Grizzle, J. M.; Curd, M. R. 1978. Posthatching histological development of the digestive system and swim bladder of logperch, Percina caprodes. Copeia, 3: 448-455.

Hoar, W. S.; Hickman, C. P. 1967. General and comparative physiology. Prentice-Hall, New Jersey, USA, 296pp.

Liem, K. F. 1980. Adaptive significance of intra and interspecific differences in the feeding repertoires of Cichlid Fishes. American Zoologist, 20: 295-314.

Maciel, C. M. R. R. 2006. Ontogenia de larvas de piracanjuba, Brycon orbignyanus Valenciennes (1849) (Characiformes, Characidae, Bryconinae). Tese de Doutorado, Universidade Federal de Viçosa, Brasil, 229pp.

Maciel Júnior, A. 1996. Desenvolvimento inicial de larvas de Curimatá-pacu (Prochilodus marggravii Walbaum, 1792) (Characiformes, Prochilodontidae), submetidas a diferentes temperaturas de incubação. Dissertação de Mestrado, Universidade Federal de Viçosa, Brasil, 60pp.

Machinandierena, L.; Muller, M.; López, A. 2003. Early life stages of development of the red porgy Pagrus pagrus (Pisces, Sparidae) in captivity, Investigaciones Marinas, 31 (1): 5-13.

Matkovic, M.; Pisanó, A. 1989. Estudio macro y microscopico del desarrollo de Hoplias malabaricus (Pisces, Erythrinidae). I: Fase larval. Revista Brasileira de Biologia, 49 (2): 553-569.

Meijide, F. J.; Guerrero, G. A. 2000. Embryonic and larval development of a substrate-brooding cichlid Cichlasoma dimerus (Heckel, 1940) under laboratory conditions. Journal of Zoology, 252: 481-493.

Menezes, N. A.; Weitzman, S. H.; Oyakawa, O. T.; Lima, F. C. T. de; Castro, R. M. C.; Weitzman, M. J. 2007. Peixes de água doce da Mata Atlântica. Lista preliminar das espécies e comentários sobre conservação de peixes de água doce neotropicais. Museu de Zoologia da Universidade de São Paulo, São Paulo, Brasil, 408pp. 
Nakatani, K.; Agostinho, A. A.; Baumgartner, G.; Bialetzki, A.; Sanches, P. V.; Makrakis, M. C.; Pavanelli, C. S. 2001. Ovos e larvas de peixes de água doce: Desenvolvimento e manual de identificação. EDUEM, Maringá, Brasil, 378pp.

Neves, C. A. 1996. Estudo morfológico e histoenzimológico do desenvolvimento ontogenético do trato digestivo de larvas e alevinos de trairão (Hoplias cf. lacerdae) e de pacamã (Lophiosilurus alexandri). Dissertação de Mestrado, Universidade Federal de Minas Gerais, Brasil, 74pp.

Oyakawa, O. T. 1993. Revisão das espécies do gênero Hoplias (grupo lacerdae) da Amazônia brasileira e região leste do Brasil (Teleostei: Erythrinidae). Resumos do $\mathbf{X}^{\mathbf{0}}$ Encontro Brasileiro de Ictiologia, São Paulo, Brasil, p.76.

Paiva, M. P. 1974. Crescimento, alimentação e reprodução de traíra, Hoplias malabaricus (Bloch), no nordeste brasileiro. Imprensa Universitária da UFC, Fortaleza, Brasil, 32pp.

Pinder, A. C.; Gozlan, R. E. 2004. Early ontogeny of sunbleak. Journal of Fish Biology, 64: 762-775.

Ricker, W. E. 1968. Methods for assessment of fish production in fresh waters. Blackwell Scientific, London, UK, 313pp.

Santos, J. E. 1992. Ontogênese e comportamento larvais de seis espécies de peixes de água doce sob condições experimentais. Dissertação de Mestrado, Universidade Federal de Minas Gerais, Brasil, 132pp.
Santos, J. E.; Godinho, H. P. 1996. Ontogênese e comportamento natatório das larvas do pacu (Piaractus mesopotamicus Homberg, 1887) mantidas experimentalmente em três regimes de fotoperíodo. BIOS, 4 (4): 11-16.

Santos, J. E.; Godinho, H. P. 2002. Ontogenic events and swimming behavior of laarvae of the characid fish Salminus brasiliensis (Cuvier) (Characiformes, Characidae) under laboratory conditions. Revista Brasileira de Zoologia, 29 (1): 163-171.

Sirol, R. N. 1995. Efeito da inanição sobre o desenvolvimento inicial da larva de Hoplias ef. lacerdae Ribeiro, 1908 (Characiformes: Erytrinidae). Dissertação de Mestrado, Universidade Federal de Viçosa, Brasil, 61pp.

Souza, S. N. 1999. Avaliação da estrutura do aparelho digestivo de alevinos de surubim, Pseudoplatystoma coruscans Agassiz, 1829 (Siluriformes, Siluroidei, Pimelodidae) relacionada com a sua capacidade de selecionar e digerir o alimento. Dissertação de Mestrado, Universidade Federal de Viçosa, Brasil, 95pp.

Walford, J.; Lam, T. J. 1993. Development of digestive tract and proteolytic enzime activity in seabass (Lates calcarifer) larvae and juveniles. Aquaculture, 109:187-205.

Woynarovich, E.; Horváth, L. 1989. A propagação artificial de peixes tropicais: Manual de extensão. Ministério da Agricultura, FAO/CODEVASF/CNPq, Brasília, Brasil, 225pp. 